\title{
Karl Gabriel
}

\section{Zur Bedeutung der Religion für Gesellschaft und Lebensführung in Deutschland}

\section{Einleitung}

Glaubte man noch vor einigen Jahren genau zu wissen, wohin es mit der Religion geht, nämlich - je nach dem Grad der gesellschaftlichen Modernisierung - zu ihrem kontinuierlichen Rückgang und Relevanzverlust, so ist die Einschätzung heute vorsichtiger, uneindeutiger und widersprüchlicher geworden ${ }^{1}$. Zwar zählt die Säkularisierung nach wie vor zu den Entwicklungen, die für die meisten Soziologen - neben Urbanisierung, Industrialisierung, Alphabetisierung etc. - zu den konstitutiven Dimensionen gesellschaftlicher Modernisierung gehören, aber es häufen sich die Zwänge, Zusatzannahmen einführen und Ausnahmen von der Regel konzidieren zu müssen. Dies ist das Feld, auf dem sich gegenwärtig der Streit um die Ablösung des Säkularisierungsparadigmas in der Religionssoziologie abspielt ${ }^{2}$. Mit der These der Privatisierung der Religion als strukturell angelegter Entwicklungsrichtung von Religiosität in der Moderne, liegt faktisch schon seit den sechziger Jahren ein theoretisch und inzwischen auch empirisch gehaltvolles Alternativkonzept zur Säkularisierung vor ${ }^{3}$. Es ist in der Diskussion der letzten Jahre in den größeren Zusammenhang des Konzepts der gesellschaftlichen Individualisierung gerückt worden. Mit einer gewissen Berechtigung läßt sich sagen, daß Luckmanns These auf dem Feld der Religion viel von dem vorweggenommen

${ }^{1}$ Einen Überblick über neuere Entwicklungen geben: Hans-Joachim Höhn (Hrsg.), Krise der Immanenz. Religion an den Grenzen der Moderne (Frankfurt a.M. 1996); Otto Kallscheuer (Hrsg.), Das Europa der Religionen. Ein Kontinent zwischen Säkularisierung und Fundamentalismus (Frankfurt a.M. 1996); Heinrich Schmidinger (Hrsg.), Religiosität am Ende der Moderne. Krise oder Aufbruch? (Innsbruck, Wien 1999); Zentrum für Europaund Nordamerika-Studien (Hrsg.), Religion und Politik. Zwischen Universalismus und Partikularismus (Jahrbuch für Europa- und Nordamerika-Studien 2, Opladen 2000).

${ }^{2}$ Karl Gabriel (Hrsg.), Religiöse Individualisierung oder Säkularisierung. Biographie und Gruppe als Bezugspunkte moderner Religiosität (Gütersloh 1996).

3 Thomas Luckmann, Die unsichtbare Religion. Mit einem Vorwort von Hubert Knoblauch (Frankfurt a.M. 1991); im folgenden zitiert: Luckmann, Religion. Das Buch geht auf eine in Freiburg i. Br. erschienene deutsche Urfassung aus dem Jahr 1963 (Das Problem der Religion in der modernen Gesellschaft) und eine in New York 1967 publizierte amerikanische Fassung (The Invisible Religion) zurück. 
hat, was die Vertreter des Individualisierungskonzepts heute als theoretische Innovation ins Spiel bringen ${ }^{4}$. Die Infragestellung des Säkularisierungskonzepts ist aber bei der These der Privatisierung bzw. Individualisierung nicht stehen geblieben. Weltweit sieht sich die Religionstheorie mit Phänomenen konfrontiert, die auf eine Tendenz zur Deprivatisierung der Religion hindeuten ${ }^{5}$. Die Hinweise reichen von den neuen Virulenzen fundamentalistischer Tendenzen in allen Weltreligionen über Anzeichen eines Wiedererstarkens öffentlicher Religion in der hochmodernisierten Gesellschaft der Vereinigten Staaten bis zum weltweiten Erfolg sogenannter Befreiungstheologien in den Ländern der Dritten Welt. So sieht sich die religionssoziologische Deutung der religiösen Entwicklung heute in das Spannungsfeld von Säkularisierung, religiöser Privatisierung und Individualisierung sowie Tendenzen einer weltweiten Deprivatisierung der Religion hineingestellt ${ }^{6}$.

Die Überwindung eines deterministischen Verständnisses im Verhältnis von Religion und Modernität im neueren Religionsdiskurs erlaubt auch einen offeneren und komplexeren Blick auf die Stellung der Religion in der deutschen Gesellschaft und ihren Einfluß auf die Lebensführung der Deutschen in der Epoche des Ost-West-Konflikts. Es gehört zu den Charakteristika dieser Epoche, daß ein verändertes Muster im Verhältnis von Religion und Modernität bzw. in der Verflechtung von Religion und Gesellschaft zum Durchbruch kommt?

Für eine vergleichende Analyse dieses Wandels bietet das Nachkriegsdeutschland eine exzeptionelle Grundlage, weil die konfessionellen Traditionen von Protestantismus und Katholizismus zusammen involviert sind und sie es gleichzeitig mit zwei divergierenden politischen Regimen mit alternativen Religionspolitiken zu tun haben. Gerade in Deutschland gingen seit der Mitte des 19. Jahrhunderts Modernisierung und Konfessionalisierung zumindest teilweise Hand in Hand. Für große Teile der katholischen Bevölkerung wurde die Konfession zu einem entscheidenden Bezugspunkt, an dem sie sich im Durchbruch zur Moderne organisierte und orientierte. Im Katholizismus mischten sich auf komplexe Weise die Merkmale einer antimodernen, restaurativen Bewegung mit Elementen religiöser, sozialer und politischer Modernisierung. ${ }^{8}$. Wenn auch ohne eine ähnliche Geschlossenheit und soziale Dichte wie der Minderheitskatholizismus entwickelte

${ }^{4}$ Constans Seyfarth, Religionssoziologische Aspekte der Wertwandlungsproblematik, in: Hans May (Hrsg.), Religion und gesellschaftlicher Wandel (Loccumer Protokolle 8, Loccum 1984) 11-38.

5 José Casanova, Public Religions in the Modern World (Chicago, London 1994).

${ }^{6} \mathrm{Karl} \mathrm{Gabriel,} \mathrm{Religionssoziologie:} \mathrm{Religion} \mathrm{zwischen} \mathrm{Säkularisierung,} \mathrm{Individualisierung}$ und Deprivatisierung, in: Richard Münch, Claudia Jauß, Carsten Stark (Hrsg.), Soziologie 2000. Kritische Bestandsaufnahme zu einer Soziologie für das 21. Jahrhunderts (Soziologische Revue Sonderheft 5, München 2000) 253-254.

7 Karl Gabriel, Tradition im Kontext enttraditionalisierter Gesellschaft, in: Dietrich Wiederkebr (Hrsg.), Wie geschieht Tradition? Überlieferung im Lebensprozeß der Kirche (Freiburg i. Br. 1991) 69-88, 77.

8 Wilfried Loth, Der Katholizismus und die Durchsetzung der Demokratie in Deutschland, in: Martin Greschat, Jochen-Christoph Kaiser (Hrsg.), Christentum und Demokratie im 20. Jahrhundert (Konfession und Gesellschaft 4, Stuttgart 1992) 111-133, 111. 
auch die protestantische Mehrheitstradition Amalgamierungen von restaurativer Beharrung und Modernisierung. Wie sich exemplarisch etwa an der spezifisch deutschen sozialpolitischen Tradition zeigen läßt, besaßen die konfessionellen Kulturen erheblichen Einfluß auf den Modernisierungspfad, den die deutsche Gesellschaft seit dem Kaiserreich einschlug'. Gleichzeitig konnten die Konfessionen mit den Mitteln moderner Bewegung und Organisation - trotz des gesellschaftlichen Durchbruchs zur Moderne - ihre Mitgliedschaft auf veränderte Weise an sich binden und stabilisieren. Das von konfessionellen und weltanschaulichen $\mathrm{Mi}$ lieus geprägte Muster gesellschaftlicher und religiös-weltanschaulicher Modernisierung in Deutschland reichte bis in die Nachkriegsphase hinein, fand aber in der Epoche des Ost-West-Konflikts ihr Ende.

Vor diesem Hintergrund soll im folgenden die Entwicklung des religiösen Faktors zunächst in der alten Bundesrepublik, sodann in der DDR zur Darstellung kommen und jeweils einer religionstheoretischen Interpretation auf der Grundlage eines modernisierungstheoretischen Interpretationsrahmens zugänglich gemacht werden ${ }^{10}$.

\section{Die Entwicklung des religiösen Faktors in der Bundesrepublik}

Das Ende des Zweiten Weltkriegs bringt einen Einschnitt für die Bedeutung der Religion in Deutschland ${ }^{11}$. Mit der Gründung der beiden deutschen Staaten trennen sich auf sehr nachdrückliche Weise auch die Wege der beiden Religionssysteme $^{12}$. In der Bundesrepublik Deutschland kommt es zum ersten Mal in der deutschen Geschichte zu einer zahlenmäßigen Parität zwischen Protestanten und Katholiken. In Folge der Flüchtlingsströme lösen sich wie nie zuvor seit dem Augsburger Religionsfrieden die konfessionell geschlossenen Siedlungsgebiete auf. In den fünfziger und frühen sechziger Jahren erhalten die Kirchen in der Bundesrepublik eine für ihre Geschichte in Deutschland einmalige Stellung. Sie repräsentieren eine gewisse institutionelle Kontinuität und gelten von allen Institutio-

9 Jochen-Christoph Kaiser, Wilfried Loth (Hrsg.), Soziale Reform im Kaiserreich. Protestantismus, Katholizismus und Sozialpolitik (Konfession und Gesellschaft 11, Stuttgart 1997) $15 \mathrm{ff}$.

${ }^{10}$ Franz-Xaver Kaufmann, Religion und Modernität. Sozialwissenschaftliche Perspektiven (Tübingen 1989).

11 Für ausführlichere Fassungen siehe: Karl Gabriel, Christentum zwischen Tradition und Postmoderne (Freiburg i. Br. ${ }^{72000)}$ 27-68; ders., Kirchen/Religionsgemeinschaften, in: Bernhard Schäfers, Wolfgang Zapf (Hrsg.), Handwörterbuch zur Gesellschaft Deutschlands (Opladen 22001) 380-391.

12 Karl-Fritz Daiber, Religion unter den Bedingungen der Moderne. Die Situation in der Bundesrepublik Deutschland (Marburg 1995); Michael N. Ebertz, Kirche im Gegenwind. Zum Umbruch in der religiösen Landschaft (Freiburg i. Br. 1997); ders., Erosion der Gnadenanstalt? Zum Wandel der Sozialgestalt von Kirche (Frankfurt a.M. 1998); Heiner Meulemann, Säkularisierung, Kirchenbindung und Religiosität, in: Bernhard Schäfers, Wolfgang Zapf (Hrsg.), Handwörterbuch zur Gesellschaft Deutschlands (Opladen 22001) 563-573. 
nen als am wenigsten durch den Nationalsozialismus kompromittiert. Weite Kreise der Bevölkerung erwarten von den Kirchen nach dem Fiasko des Nationalsozialismus Orientierung und Wertevermittlung. Die Kirchenbesucherzahlen sind in beiden Konfessionen - gemessen jeweils an ihren Traditionen - hoch und steigen innerhalb der fünfziger Jahre sogar leicht an. Auch die Jugendlichen sind überdurchschnittlich in die Gottesdienstpraxis integriert. Die Austritte aus beiden Kirchen sind auf einem historisch einmalig niedrigem Niveau und spielen so gut wie keine Rolle. Was für die Kirchenmitgliedschaft gilt, läßt sich auch für die Riten an den Lebenswenden konstatieren: Bis auf geringe Ausnahmen ist die gesamte Bevölkerung in sie einbezogen. Die Kirchen erhalten eine privilegierte verfassungsrechtliche Stellung wie kaum anderswo in der Welt. Die Übernahme der Kirchenartikel der Weimarer Verfassung in das Grundgesetz ermöglicht den staatlichen Einzug der Kirchensteuer. Weite Teile des staatlichen Schulsystems bleiben bzw. erhalten eine kirchlich-konfessionelle Prägung. Der kirchlich kontrollierte Religionsunterricht wird als Pflichtfach in allen Schulformen institutionalisiert. Die Sozialgesetzgebung der Bundesrepublik räumt den freien und das heißt dominierend den kirchlichen Trägern der Wohlfahrtspflege - dem Subsidiaritätsprinzip der katholischen Soziallehre folgend - einen prinzipiellen Vorrang vor den öffentlichen Trägern ein. Darin kommt der große Einfluß der kirchlichen Soziallehren insbesondere der katholischen - auf die staatliche Sozialpolitik in dieser ersten Phase der Geschichte der Bundesrepublik zum Ausdruck. Wie die Forschung zum Wertewandel hervorhebt, dominiert in der Bundesrepublik eine konservativere Grundstimmung mit einer Bevorzugung traditioneller Pflicht- und Akzeptanzwerte ${ }^{13}$.

In der Latenz schon länger vorbereitet, vollzieht sich in der Gesellschaft der Bundesrepublik Ende der sechziger Jahre ein tiefgreifender Umbruch. Vom strukturellen wie kulturellen Modernisierungsschub, der Mitte der 60er Jahre einsetzt, zeigen sich die beiden Kirchen in besonders nachhaltiger Weise betroffen. In dem knappen Zeitraum zwischen 1968 und 1973 gehen - als Indikator für weiterreichende Bewußtseins- und Verhaltensänderungen - die Kirchenbesucherzahlen sprunghaft um ca. ein Drittel zurück. Bei den jüngeren Jahrgängen macht der Rückgang sogar knapp die Hälfte aus. Die Kirchenaustritte schnellen um ein Vielfaches nach oben und verweisen auf eine Lockerung der Kirchenbindung. Die Bildungsreform beseitigt weitgehend die konfessionelle Prägung des staatlichöffentlichen Schulsystems. Der kirchliche Einfluß auf die Politik geht deutlich zurück. Erste größere kirchensoziologische Untersuchungen machen auf eine gewachsene Distanz der Gläubigen zu den kirchlichen Lehren und Verhaltensnormen aufmerksam ${ }^{14}$. Der kirchliche Wandel zeigt sich eingebettet in einen Wert-

13 Helmut Klages, Wertorientierungen im Wandel. Rückblick, Gegenwartsanalyse, Prognosen (Frankfurt a.M. 21985); ders., Traditionsbruch als Herausforderung. Perspektiven der Wertewandlungsgesellschaft (Frankfurt a.M. 1993); im folgenden zitiert: Klages, Traditionsbruch.

14 Gerhard Schmidtchen, Zwischen Kirche und Gesellschaft. Forschungsbericht über die Umfragen zur Gemeinsamen Synode der Bistümer der Bundesrepublik Deutschland (Frei- 
wandlungsschub von der Dominanz von persönlichen wie gesellschaftlichen Pflicht- und Akzeptanzwerten hin zu einem Bedeutungszuwachs von Selbstentfaltungswerten. Vom gesellschaftlichen Umbruch sind beide konfessionellen volkskirchlichen Systeme in ähnlicher Weise tangiert. Allerdings trifft die Erosion der bis in das 19. Jahrhundert zurückreichenden sozial-moralischen Milieus den Katholizismus schärfer als den Protestantismus. Neben der sozialistischen Arbeiterschaft besaßen die Katholiken das sozial-moralische Milieu mit der stärksten Bindungskraft ${ }^{15}$. Der abrupte Anstieg des Lebens- wie des Bildungsniveaus unter den Katholiken setzt den Erosionsprozeß in Gang. Verbunden mit dem Ausbau der sozialen Sicherungssysteme, der Ausweitung der arbeitsfreien Zeit, der Revolution in der Motorisierung und im Mediengebrauch lassen diese Faktoren eine stärker individualisierte Lebensführung auch unter den Katholiken entstehen ${ }^{16}$.

Ende der siebziger und in den achtziger Jahren verlangsamt sich das Tempo des sozialen und religiösen Wandels in der Bundesrepublik wiederum deutlich. Die Kirchenaustrittszahlen bewegen sich von ihrem Höhepunkt 1973/74 nach unten, bevor sie gegen Ende des Jahrzehnts erneut nach oben schnellen. Auch die Kirchenbesucherzahlen sinken zwar stetig weiter, aber nicht mehr mit dem Tempo der frühen siebziger Jahre. In beiden volkskirchlichen Systemen geht der Prozeß der Differenzierung der Mitgliedschafts- und Teilnahmeformen weiter. Als stabilstes Element von Kirchlichkeit erweisen sich die Teilnahmeformen an den kirchlichen Ritualen zu den Lebenswenden. Bis auf gewisse Erosionstendenzen in den Ballungszentren bleibt das kirchliche Ritenmonopol an den Lebenswenden erhalten. Eine wachsende Mehrheit von Kirchenmitgliedern greift fallweise und bei Gelegenheit auf die Begleitung und Unterstützung durch die kirchliche Religion zurück, nutzt im übrigen aber die kirchlichen Orientierungsangebote als Material für den „Flickenteppich“ individualisierter, gewissermaßen selbst komponierter Religiosität. Auf der anderen Seite stabilisiert sich um die Gemeinden und den regelmäßigen sonntäglichen Kirchenbesuch herum eine kirchlich geprägte Religiosität mit Wirkung auf Wertorientierungen und Solidarverhalten. Allerdings zeigt die Kirchlichkeit eine ausgeprägte Tendenz zur Überalterung, so daß sie in hohem Maße zum Ausdruck einer Alterskultur zu werden scheint.

burg i. Br. 1972); ders., Gottesdienst in einer rationalen Welt. Religionssoziologische Untersuchungen im Bereich der VELKD (Stuttgart, Freiburg 1973); Helmut Hild (Hrsg.), Wie stabil ist die Kirche? (Gelnhausen, Berlin 1974).

15 Josef Mooser, Auflösung der proletarischen Milieus. Klassenbindung und Individualisierung in der Arbeiterschaft vom Kaiserreich bis in die Bundesrepublik Deutschland, in: Soziale Welt 34 (1983) 270-306: Wilhelm Damberg, Abschied vom Milieu? Katholizismus im Bistum Münster und in den Niederlanden 1945-1980 (Paderborn 1997); Karl Gabriel, Zwischen Aufbruch und Absturz in die Moderne. Die katholische Kirche in den 60er Jahren, in: Axel Schildt, Detlef Siegfried, Karl Christian Lammers (Hrsg.), Dynamische Zeiten. Die 60er Jahre in den beiden deutschen Gesellschaften (Hamburg 2000) 528-543.

${ }_{16}$ Helmut Klages, Traditionsbruch 62-65. 


\section{Religion als gesellschaftliche Gestaltungskraft: Kontinuität und Wandel}

Wenn auch die Entwicklung der Bundesrepublik seit Mitte der 60er Jahre auf den ersten Blick durch einen weitreichenden Einflußverlust der Religion auf Gesellschaft und Politik gekennzeichnet erscheint, so lassen sich bei näherer Betrachtung doch auch gewichtige Kontinuitäten ausmachen. $\mathrm{Zu}$ den überraschendsten Phänomenen gehört, daß der konfessionell-religiöse Einfluß auf das Wahlverhalten der Bundesbürger weitgehend erhalten bleibt ${ }^{17}$. Die bis auf Spannungslinien des Kaiserreichs im Jahrzehnt des Kulturkampfs zurückgehende Wahlpräferenz der Katholiken für die CDU übersteht die Erosionstendenzen des katholischen Milieus wie den Umbruch in der Kirchenbindung der Katholiken. Zwischen 1953 und 1982 sinkt zwar der Anteil der regelmäßigen Kirchgänger unter den Katholiken von $61 \%$ auf $31 \%$ und steigt der Anteil der Selten/Nie-Kirchgänger von $8 \%$ auf $34 \%$. Im gleichen Zeitraum nimmt aber die Wahlpräferenz der Katholiken für die CDU insgesamt um 3\% - von $57 \%$ auf $60 \%$ - zu, wobei alle Gruppen der Kirchgangshäufigkeit Steigerungsraten aufweisen ${ }^{18}$. Karl Schmitt erklärt die erstaunliche Kontinuität der Wahlpräferenz der Katholiken in der Bundesrepublik durch einen Transfer der Parteienbindung von einer „sozial abgeleiteten“ in eine „originäre" CDU/CSU-Parteibindung, die insbesondere durch die Entscheidung gegen die Wiedererrichtung des Zentrums und für eine interkonfessionelle Partei 1945 erleichtert wurde ${ }^{19}$. Seine These einer im Generationenwechsel verspäteten Entkonfessionalisierung des Wählerverhaltens der Katholiken kann sich durch die jüngsten Wahlergebnisse bestätigt sehen ${ }^{20}$.

Hohe Kontinuität mit wachsender Tendenz weist auch der Einfluß der Kirchen auf das expandierende Feld der sozialen bzw. personenbezogenen Dienstleistungen auf. Wie der Personalstatistik des Deutschen Caritasverbands zu entnehmen

${ }^{17}$ Franz-Urban Pappi, Sozialstruktur, gesellschaftliche Wertorientierungen und Wahlabsicht. Politische Vierteljahresschrift 18 (1977) 195-229; ders., Konstanz und Wandel der Hauptspannungslinien in der Bundesrepublik, in: Joachim Matthes (Hrsg.), Sozialer Wandel in Westeuropa (Frankfurt a.M. 1979) 465-479; ders., Die konfessionell-religöse Konfliktlinie in der deutschen Wählerschaft: Entstehung, Stabilität und Wandel, in: Dieter Oberndörfer, Hans Rattinger, Karl Schmitt (Hrsg.), Wirtschaftlicher Wandel, religiöser Wandel und Wertwandel: Folgen für das politische Verhalten in der Bundesrepublik Deutschland (Berlin 1985) 263-290; Karl Schmitt, Religiöse Bestimmungsfaktoren des Wahlverhaltens: Entkonfessionalisierung mit Verspätung?, in: Dieter Oberndörfer, Hans Rattinger, Karl Schmitt (Hrsg.), Wirtschaftlicher Wandel, religiöser Wandel und Wertwandel: Folgen für das politische Verhalten in der Bundesrepublik Deutschland (Berlin 1985) 291-329; Karl Schmitt, Konfession und Wahlverhalten in der Bundesrepublik Deutschland (Berlin 1989).

18 Karl Schmidt, Religiöse Bestimmungsfaktoren 292.

19 Karl Schmidt, Religiöse Bestimmungsfaktoren 326.

$20 \mathrm{Jörg} \mathrm{Jacobs,} \mathrm{Die} \mathrm{konfessionell-religiöse} \mathrm{Spannungslinie} \mathrm{am} \mathrm{Beispiel} \mathrm{der} \mathrm{Bundestagswahlen}$ 1994 und 1998, in: Detlef Pollack, Gert Pickel (Hrsg.), Religiöser und kirchlicher Wandel in Ostdeutschland 1989-1999 (Opladen 2000) 165-185; Karl Gabriel, Zur gesellschaftlichen Präsenz der Katholiken, in: Wolfgang Thierse (Hrsg.), Religion ist keine Privatsache (Düsseldorf 2000) 120-132, 122. 
ist, verzeichnet die verbandliche Caritas in der Bundesrepublik in den sechziger und siebziger Jahren jeweils Zuwachsraten ihres Personalbestands von $39 \%$ bzw. $48 \%{ }^{21}$. Im gleichen Zeitraum geht die Zahl der Ordensangehörigen unter den hauptberuflich Tätigen der Caritas von 58000 auf 33000 zurück, was einen weitreichenden Wandel in der Personalstruktur anzeigt. Unbeschadet dessen bauen Caritas und Diakonie ihre Stellung als größte und einflußreichste Spitzenverbände der Freien Wohlfahrtspflege in der Bundesrepublik aus. Ihr Bedeutungszuwachs kommt auch darin zum Ausdruck, daß sie zu den größten privaten Arbeitgebern zu rechnen sind 22 . Es gelingt ihnen, in der Jugend- und Sozialhilfegesetzgebung des Jahres 1961 die eigene Stellung mit Hilfe des Subsidiaritätsprinzips zu sichern und den weiteren Ausbau der sozialen Dienste in den sechziger und siebziger Jahren maßgeblich mitzugestalten. Auch hier reicht die Kontinuität vom Beginn einer „dualen“ Wohlfahrtspflege im Kaiserreich über die Institutionalisierung der Spitzenverbände der Freien Wohlfahrtspflege im Weimarer Sozialstaat bis in die sozialstaatliche Entwicklung der Bundesrepublik hinein ${ }^{23}$.

Der Einfluß des religiösen Faktors spiegelt sich auch in der Kontinuität spezifischer Konfliktarenen wider, die der Bundesrepublik ihr Gepräge geben. In den frühen Jahren sind es die konfessionellen wie innerkirchlichen Konfliktstoff freisetzenden Auseinandersetzungen um die Wiederbewaffnung, den Wehrdienst und die Militärseelsorge sowie die atomare Wiederaufrüstung der Bundeswehr ${ }^{24}$. Zu den zentralen Konfliktarenen gehört auch die Auseinandersetzung um den $\$ 218$, die mit den Neuregelungsversuchen seit 1974 eskaliert und bis in die Gegenwart hinein andauert ${ }^{25}$. In der kirchlicherseits traditionell mit hohem Gewicht versehenen Arena der Schulpolitik verlieren die Bischöfe in der Verteidigung des konfessionellen Charakters des öffentlichen Schulwesens den Rückhalt in der eigenen Mitgliedschaft und müssen eine empfindliche Niederlage hinnehmen ${ }^{26}$. Was den

21 Thomas Rauschenbach, Matthias Schilling, Die Dienstleistenden. Wachstum, Wandel und wirtschaftliche Bedeutung des Personals in Wohlfahrts- und Jugendverbänden, in: ders., Christoph Sachße, Thomas Olk (Hrsg.), Von der Wertgmeinschaft zum Dienstleistungsunternehmen. Jugend- und Wohlfahrtsverbände im Umbruch (Frankfurt a.M. 1995) 321-355; Karl Gabriel, Caritas angesichts fortschreitender Säkularisierung, in: Erwin Gatz (Hrsg.), Geschichte des kirchlichen Lebens in den deutschsprachigen Ländern seit dem Ende des 18. Jahrhunderts (Band V: Caritas und soziale Dienste, Freiburg, Basel, Wien 1997) 438-455.

22 Rauschenbach, Schilling, Die Dienstleistenden $338 \mathrm{ff}$.

${ }^{23}$ Christoph Sachße, Verein, Verband und Wohlfahrtsstaat: Entstehung und Entwicklung der "dualen" Wohlfahrtspflege, in: Thomas Rauschenbach, Christoph Sachße, Thomas Olk (Hrsg.), Von der Wertgmeinschaft zum Dienstleistungsunternehmen. Jugend- und Wohlfahrtsverbände im Umbruch (Frankfurt a.M. 1995) 123-149; Karl Gabriel, Die religiösen Wurzeln von "dualer" Wohlfahrtspflege und Wohlfahrtsstaat in Deutschland. Das Beispiel des Katholizismus, in: Zentrum für Europa- und Nordamerika-Studien (Hrsg.), Religion und Politik. Zwischen Universalismus und Partikularismus (Opladen 2000) 203-224.

${ }_{24}$ Anselm Doering-Manteuffel, Katholizismus und Wiederbewaffnung. Die Haltung der deutschen Katholiken gegenüber der Wehrfrage 1948-1955 (Mainz 1981).

${ }_{25}$ Michael Gante, $\$ 218$ in der Diskussion. Meinungs- und Willenbildung 1945-1976 (Düsscldorf 1991); Jobannes Reiter, Rolf Keller (Hrsg.), Herausforderung Schwangerschaftsabbruch. Fakten, Argumente, Perspektiven (Freiburg i. Br. 1992).

26 Frederic Spotts, Kirchen und Politik in Deutschland (Stuttgart 1973) $191 \mathrm{ff}$. 
kirchlich verantworteten Religionsunterricht als ordentliches Schulfach in allen öffentlichen Schulformen angeht, enden die immer wieder aufbrechenden öffentlichen Diskurse - ähnlich wie in der Frage des staatlichen Einzugs der Kirchensteuer - mit einer Festigung der kirchlichen Position ${ }^{27}$.

Eng mit den angesprochenen Kontinuitätslinien verbunden nimmt der religiöskirchliche Einfluß auf die Gesellschaft aber auch veränderte Formen an. Mit weitreichenden Folgen für die gesellschaftliche Entwicklung in der Bundesrepublik überwinden beide Kirchen ihre traditionelle Ambivalenz gegenüber Demokratie und Pluralismus ${ }^{28}$. Dominieren im ersten Jahrzehnt noch Hoffnungen und Programme einer umfassenden Rechristianisierung und Vorstellungen eines hierarchischen, gesellschaftsenthobenen Wächteramts der Kirchen die gesellschaftliche Orientierung, so bekommt in der Folgezeit die Demokratie als Staats- und Lebensform wachsenden Rückhalt in den kirchlichen und konfessionellen Kulturen. Mit der Erklärung zur Religionsfreiheit und der Pastoralkonstitution "Gaudium et spes" kommt im Katholizismus ein neues Verhältnis zu Demokratie und Öffentlichkeit zum Durchbruch ${ }^{29}$.

Wenn es im Protestantismus auch bis zum Jahr 1985 dauert, ehe die Kammer für öffentliche Verantwortung mit der Demokratie-Denkschrift der EKD einen Schlußstrich unter protestantische Ambivalenzen gegenüber der Demokratie zieht, so kommt in ihr nur eine schon länger gefestigte kirchliche Praxis zum Ausdruck. Im Protestantismus initiiert und vom Katholizismus früh übernommen, werden die kirchlichen Akademien nicht nur zum Raum einer neuen, dauerreflexiven Sozialform des Glaubens ${ }^{30}$, sondern auch zu wirksamen Drehscheiben wechselseitiger Einflußprozesse von Kirche und Gesellschaft ${ }^{31}$. Akademiediskurse begründen auch eine besondere Nähe und Verantwortung der Kirchen zur bundesrepublikanischen Wirtschaftsordnung der sozialen Marktwirtschaft mit der konstitutiven Rolle eines starken Sozialstaats. Die Diskussionskultur der Akademien, der Kirchen, aber auch der neue Formen annehmenden Katholikentage gibt auch der Öffnung der deutschen Politik für Europa, für die Versöhnung im Osten, für Menschenrechtsfragen sowie innerhalb der Entwicklungspolitik Rück-

27 Hans Maier, Schriften zu Kirche und Gesellschaft. Bd. 3: Religion und moderne Gescllschaft (Freiburg i. Br. 1985) 90-131.

28 Wilfried Loth, Der Katholizismus und die Durchsetzung der Demokratic in Deutschland, in: Martin Greschat, Jochen-Christoph Kaiser (Hrsg.), Christentum und Demokratic in Deutschland (Konfession und Gesellschaft 4, Stuttgart 1992) 111-128, 18; Kurt Nowak, Protestantismus und Demokratic in Deutschland. Aspekte der politischen Moderne, in: Greschat, Kaiser (Hrsg.), Christentum 1-18, 10-13.

29 Ernst-Wolfgang Böckenförde, Religionsfrcihcit. Die Kirche in der modernen Wclt (Schriften zu Staat - Gesellschaft - Kirche 3, Freiburg 1990).

30 Helmut Schelsky, Ist die Dauerreflexion institutionalisierbar? Zum Thema cincr modernen Religionssoziologie, in: Zeitschrift für Evangelische Ethik 1 (1957) 153-174.

31 Rulf Jürgen Treidel, Evangelische Akademien im Nachkriegsprotestantismus: Bemerkungen zu Kontinuitäten und Wandlungen von sozialethischen Leitbildern, in: Thomas Sauer (Hrsg.), Katholiken und Protestanten in den Aufbaujahren der Bundesrepublik (Konfession und Gesellschaft 21, Stuttgart 2000) 108-120. 
halt ${ }^{32}$. Die Kirchen ziehen - so läßt sich zusammenfassen - nach einer Zwischenphase der Hoffnung auf ein "rechristianisiertes“ Deutschland und Abendland nachhaltig wirksame Lehren aus ihren Erfahrungen mit dem totalitären Staat, überwinden traditionelle Vorbehalte gegenüber der Demokratie und nehmen neue Rollen als öffentliche Akteure jenseits traditioneller, an Wirksamkeit einbüBender Konfessionspolitik wahr.

\section{Zur religionstheoretischen Interpretation}

Die religionssoziologische Theoriedebatte in Deutschland war lange Zeit durch die von Thomas Luckmann bereits in den sechziger Jahren vorgetragene These geprägt, die Kirchen repräsentierten in modernen Gesellschaften nicht mehr die dominierende Sozialform der Religion. Für differenzierte, durch rationale Institutionsgefüge und privatisierte Lebenswelten geprägte moderne Gesellschaften sei vielmehr eine individualisierte, sozial eher "unsichtbare" Form der Religion typisch ${ }^{33}$. Es entsprach der Logik des Arguments Luckmanns, wenn in der Folgezeit die Kirchensoziologie - parallel zur Marginalisierung ihres Gegenstands innerhalb der Soziologie eine Randstellung mit einem defizitären theoretischen Status zugesprochen erhielt ${ }^{34}$. Inzwischen haben sich die Fronten zwischen einer allein am Zähl- und Meßbaren interessierten Kirchensoziologie und einer Religionssoziologie auf der Suche nach der „unsichtbaren Religion“ deutlich eingeebnet. Auf der einen Seite ist es zum kirchen- und religionssoziologischen Allgemeingut geworden, den Zusammenhang von Kirchlichkeit und Religiosität als ein kontingentes Forschungsproblem zu betrachten und Religion nicht am kirchlichinstitutionell Zurechenbaren enden zu lassen. Auf der anderen Seite sind die Grenzen eines Religionsbegriffs deutlich geworden, der den gesellschaftlich-historisch gegebenen Formen der Religion - theoretisch vorentschieden - einen marginalen Status zuzuschreiben geneigt macht. Auf dieser Linie liegt es, sowohl von einer theoretisch postulierten und vorentschiedenen Marginalität der Kirchen als auch von einer prinzipiellen Gegenläufigkeit von Religiosität und Kirchlichkeit für moderne Gesellschaften Abschied zu nehmen. Religion liegt in Deutschland nach wie vor - so läßt sich resümieren - zunächst und zuerst in ihren institutionalisierten und heute zunehmend organisierten Sozialformen vor. In der Ge-

32 Traugott Jähnichen, Der Protestantismus als ,soziales Gewissen ‘ der Gesellschaft - Impulse des Deutschen Evangelischen Kirchentages zur Ausgestaltung der Sozialen Marktwirtschaft in der Ära Adenauer, in: Sauer (Hrsg.), Katholiken und Protestanten 89-107; Martin Greschat, Wilfried Lob(Hrsg.), Die Christen und die Entstehung der Europäischen Gemeinschaft (Konfession und Gesellschaft 5, Stuttgart 1994); Katharina Kunter, Die Kirchen im KSZE-Prozeß 1968-1978 (Konfession und Gesellschaft 20, Stuttgart 2000).

${ }^{33}$ Luckmann, Religion 178-183.

${ }^{34}$ Andreas Feige, Kirchenmitgliedschaft in der Bundesrepublik Deutschland. Zentrale Perspektiven empirischer Forschungsarbeiten im problemgeschichtlichen Kontext der deutschen Religions- und Kirchensoziologie nach 1945 (Gütersloh 1990). 
samtbetrachtung wird man die Kirchen und die kirchlich verfaßte Religion in der Bundesrepublik - so ist deutlich geworden - auch nicht als marginale Größen betrachten können. Dazu reicht ihr vielfältiger Einfluß viel zu weit in die öffentlichen Zonen des gesellschaftlichen Lebens hinein. Auf der anderen Seite sind längerfristige Prozesse der Individualisierung religiöser Erfahrungen unverkennbar. Sie reichen bis in Kernbereiche der Großkirchen hinein und zeichnen dafür verantwortlich, daß die gerade in der bundesrepublikanischen Geschichte außerordentlich hohe religiöse Bindungs- und Integrationskraft der Kirchen sinkt und Kirchlichkeit und Religiosität tatsächlich schärfer auseinandertreten.

\section{Entwicklung von Religion und Kirche in der DDR}

Die Entwicklung in der DDR verläuft mit einer gewissen Spiegelbildlichkeit zu der in der Bundesrepublik ${ }^{35}$. Im Jahr 1950 sind von den 18,3 Mio. Einwohnern der DDR 14,8 Mio. evangelisch und 1,37 Mio. katholisch. Das letzte statistische Jahrbuch der DDR aus dem Jahr 1990 zählt 5,1 Mio. Mitglieder der evangelischen Kirche und 1,1 Mio. der katholischen Kirche ${ }^{36}$. Die Kehrseite der kirchlichen Entwicklung zeigt sich exemplarisch an folgenden Zahlen: Wie das „Handbuch der Jugendweihe“ ausweist, nehmen seit 1976 etwa 97\% eines Jahrgangs an der atheistisch geprägten Jugendweihe teil ${ }^{37}$. Bis 1990 zeigt sich die mehrheitlich atheistisch geprägte Position der Jugend durch den politischen Umbruch wenig beeinflußt. So bezeichnen sich noch 1989 85\% der Studenten der DDR als atheistisch und nur $6 \%$ als religiös ${ }^{38}$.

Bestimmend für die Lage von Religion und Kirche wird - als Kontinuitätslinie durch die vierzigjährige Geschichte der DDR sich hindurchziehend - die mit allen Mitteln staatlicher Macht betriebene Zurückdrängung und Verbannung der Kirchen aus der gesellschaftlichen Öffentlichkeit und die stetigen Versuche der Spal-

${ }_{35}$ Hierzu und zum Folgenden siehe: Detlef Pollack, Kirche in der Organisationsgesellschaft. Zum Wandel der gesellschaftlichen Lage der evangelischen Kirchen in der DDR (Stuttgart, Berlin, Köln 1994); im folgenden zitiert: Pollack, Kirche; Detlef Pollack, Irena Borowik, Wolfgang Jagodzinski (Hrsg.), Religiöser Wandel in den postkommunistischen Ländern Ostund Mitteleuropas (Würzburg 1998); Detlef Pollack, Gert Pickel (Hrsg.), Religiöser und kirchlicher Wandel in Ostdeutschland 1989-1999 (Opladen 2000); Wolf-Jürgen Grabner, Detlef Pollack, Jugend und Religion in Ostdeutschland, in: Karl Gabriel, Hans Hobelsberger (Hrsg.), Jugend, Religion und Modernisierung. Kirchliche Jugendarbeit als Suchbewegung (Opladen 1994) 91-116; Klaus Hartmann, Detlef Pollack, Gegen den Strom: Kircheneintritte in Ostdeutschland nach der Wende (Opladen 1998); Kurt Nowak, Historische Wurzeln der Entkirchlichung in der DDR, in: Heinz Sabner, Stefan Scbwendtner (Hrsg.), Gesellschaft im Umbruch: 27. Kongreß der Deutschen Gesellschaft für Soziologie (Kongreßband II, Opladen 1995) 665-669.

36 Statistisches Jahrbuch der DDR (Berlin $1955 \mathrm{ff}$.).

37 Herbert Reitinger, Die Rolle der Kirche im politischen Prozeß der DDR 1970 bis 1990 (München 1991) 34.

38 Walter Friedrich, Mentalitätswandlungen der Jugend in der DDR, in: Aus Politik und Zeitgeschichte B 16-17 (1990) 27. 
tung zwischen Kirchenleitungen und Kirchenvolk einerseits und zwischen - im Sinne der Staatsmacht - „fortschrittlichen“ und „rückschrittlichen“ Kräften in den Kirchen andererseits. Dem staatlich-ideologischen Homogenisierungsdruck erweist sich - so ist insgesamt festzustellen - die anstaltlich und obrigkeitlich geprägte Kirchlichkeit weiter Teile insbesondere der evangelischen Mehrheitsbevölkerung als nicht gewachsen. Der Rückgang der Kirchenmitgliedschaft in der evangelischen Kirche erfolgt nicht kontinuierlich, sondern weist zwei Höhepunkte auf: die zweite Hälfte der 50er Jahre und die Jahre zwischen 1967 und 1975. Im Kampf um die Jugendweihe unterliegt die evangelische Kirche der Staatsmacht eindeutig. Als in der zweiten Hälfte der fünfziger Jahre die Hoffnung auf eine schnelle Wiedervereinigung schwindet, bricht unter massivem staatlichen Druck binnen weniger Jahre die volkskirchliche Konfirmationspraxis faktisch zusammen. Zur gleichen Zeit sinkt die Taufbereitschaft evangelischer Eltern rapide. Während 1950 noch ca. $90 \%$ der Kinder evangelischer Eltern getauft werden, fällt die Taufrate - der Anteil der evangelisch Getauften an der Zahl der Geburten - bis 1965 auf knapp 30\% ab. Nicht nur der Austritt, sondern auch in starkem Maße der unterlassene Eintritt reduziert die Zahl der Kirchenmitglieder bis in die achtziger Jahre hinein ${ }^{39}$. Soweit Daten zum Kirchenbesuch zur Verfügung stehen, zeigen sie schon für Mitte der fünfziger Jahre einen nur geringen Anteil von 3,8\%, der für die sechziger und siebziger Jahre um etwa einen Prozentpunkt absinkt, um in den achtziger Jahren sogar zuzunehmen ${ }^{40}$. Dabei fällt auf, daß - anders als in der Bundesrepublik seit den späten sechziger Jahren - die Jugendlichen und jungen Erwachsenen überproportional zu den regelmäßigen Kirchenbesuchern zählen.

Es ist eine Strategie der Schadensbegrenzung, die die evangelischen Kirchenleitungen - geschockt durch die Austrittswellen der 50er und 70er Jahren - den Ausgleich mit der staatlichen Macht suchen läßt. Wie an der kirchlichen Entwicklung ablesbar ist, haben die Kirchenleitungen mit dieser Strategie einen gewissen Erfolg. Es kommt seit den achtziger Jahren zu einer Stabilisierung auf geringem Niveau und mit insgesamt marginaler gesellschaftlicher Stellung.

In einer minoritären Randstellung hat sich die katholische Kirche in der SBZ/ DDR von Anfang an eingerichtet ${ }^{41}$. Verdoppelt sich durch die Flüchtlingsströme zwischen 1945 und 1949 die Zahl der Katholiken, so geht sie in den Jahren zwischen der Gründung der DDR 1949 und dem Mauerbau 1961 wiederum kontinuierlich zurück. Ihre Verlustzahlen an Mitgliedern sind insgesamt geringer als die der evangelischen Kirche, wenn auch die Katholiken an der "Sowjetzonenflucht“ überproportional beteiligt sind und der Ausfall von Taufen wie der Kirchenaus-

${ }^{39}$ Pollack, Kirche $384 \mathrm{ff}$.

40 Pollack, Kirche $406 \mathrm{ff}$.

${ }^{4}$ Josef Pilvousek, Die katholische Kirche in der DDR, in: Erwin Gatz (Hrsg.), Mittel-, West- und Nordeuropa: Kirche und Katholizismus seit 1945 (Paderborn 1998) 132-150; Martin Höllen, Loyale Distanz? Katholizismus und Kirchenpolitik in SBZ und DDR. Ein historischer Überblick in Dokumenten, Bd. 1-3 (Berlin 2000); Bernd Schäfer, Staat und katholische Kirche in der DDR (Köln, Weimar, Wien 21999). 
tritt ihre Zahl weiter reduziert. Der spezifische Charakter der katholischen Kirche als Kirche von Vertriebenen und Übersiedlern trägt dazu bei, daß sich die Katholiken von Beginn an stärker aus dem gesellschaftlichen und politischen Leben zurückziehen. Dies gilt in besonderem Maße für die „Ära Bengsch“ von 1961 bis 1979, in der Kardinal Bengsch die Wahrung der Einheit und Geschlossenheit der katholischen Kirche nach außen wie nach innen mit hoher Priorität verfolgt und erfolgreich durchsetzt. So nimmt die Dresdner Pastoralsynode von 1972-1975 in ihren Beschlüssen zwar Impulse des 2. Vatikanischen Konzils auf, insbesondere der Pastoralkonstitution "Gaudium et spes“, an einer konsequenten Umsetzung der Beschlüsse erweisen sich die Kirchenleitungen im Anschluß aber nicht sonderlich interessiert ${ }^{42}$. Die katholische Kirche ist so stets in Gefahr, ihre höhere Stabilität und Einheit nach innen mit einer noch ausgeprägteren gesellschaftlichen Isolierung als auf protestantischer Seite zu bezahlen.

Vergleicht man die Kirchenpolitik der SED mit der anderer kommunistischer Staaten - etwa der benachbarten Tschechoslowakei -, so erscheint sie - bis auf wenige Jahre eines offenen und scharfen Kirchenkampfes - eher versteckt und moderat. Dies kommt auch darin zum Ausdruck, daß die DDR den spezifisch deutschen Pfad eines "dualen“ Wohlfahrtsstaats mit einer Beteiligung der Kirchen an den personenbezogenen Dienstleistungen nicht gänzlich verläßt. So machen Anfang der achtziger Jahre die Einrichtungen der beiden Kirchen insgesamt 47\% aller Plätze in der Rehabilitation Schwerstbehinderter, 4,7\% der Krankenhäuser und 7,5\% der Plätze in den Alters- und Pflegeheimen aus ${ }^{43}$. Allein die Diakonie in der DDR verfügt 1969 über 59 Krankenhäuser und Heilstätten, 97 Heime für geistig und körperlich Behinderte, 326 Alters- und Pflegeheime, 323 Kindertagesstätten und 621 Gemeindepflegestationen, deren Zahl sich - bis auf den erheblichen, durch Nachwuchsmangel bedingten Rückgang der Gemeindepflegestationen bis 1989 nur geringfügig reduziert ${ }^{44}$. Hat man den gleichzeitigen Auszug weiter Teile der Bevölkerung aus der Kirchenmitgliedschaft im Blick, so kann es nicht überraschen, daß die Arbeit der Diakonie von Problemen innerer Säkularisierung begleitet ist. Dies gilt auch für die besonders prekäre Gratwanderung der Diakonie zwischen Kooperation und Verweigerung gegenüber der staatlichen Sozialpolitik und ihren Organen. Gemessen an der Minderheitenposition der Katholiken behält auch die Caritas in der DDR eine überraschend starke Stellung ${ }^{45}$. Eine Arbeit in verbandlichen Strukturen wie in der Bundesrepublik ist zwar nicht mög-

42 Rolf Schumacher, Kirche und sozialistische Welt. Eine Untersuchung zur Frage der Rezeption von "Gaudium et spes" durch die Pastoralsynode der katholischen Kirche in der DDR (Leipzig 1998) 227-241.

${ }^{43}$ Magdalena Heider, Die schwierige Rolle der Diakonie, in: Horst Däbn (Hrsg.), Die Rolle der Kirchen in der DDR. Eine erste Bilanz (München 1993) 189-197, 190.

${ }^{44}$ Heider, 190.

45 Ursula Pruß, Caritas in der DDR, in: Horst Dähn (Hrsg.), Die Rolle der Kirchen in der DDR. Eine crste Bilanz (München 1993) 198-212; Gerbard Lange, Ursula Pruß, Caritas in der DDR, in: Erwin Gatz (Hrsg.), Geschichte des kirchlichen Lebens in den deutschsprachigen Ländern seit dem Ende des 18. Jahrhunderts (Band V: Caritas und soziale Dienste, Freiburg, Basel, Wien 1997) 343-377. 
lich, trotzdem unterhalten unterschiedliche katholische Träger Mitte der siebziger Jahre 34 Krankenhäuser, 30 Kinder- und Säuglingsheime, 14 Heime für geistig Behinderte, 104 Alten- und Pflegeheime, ca. 140 allein von der Kirche finanzierte Kindergärten, 137 Gemeindeschwesternstationen mit stark sinkender Tendenz sowie 30 Erholungs- und Kurheime. Die Ausbildung des Personals erfolgt zum größten Teil in eigenen Ausbildungseinrichtungen. Im Bereich der Krankenpflegeausbildung kommt es 1975 zum Abschluß der einzigen formellen Vereinbarung, die es zwischen DDR-Regierung und katholischer Kirche während der gesamten DDR-Zeit überhaupt gegeben hat ${ }^{46}$.

Auf dem Feld von Diakonie und Caritas wird besonders nachdrücklich deutlich, daß die Kirchen - unbeschadet aller Tendenzen hin zu eigenständigen kirchlichen Organisationsstrukturen - eine besondere Funktion der Klammer zwischen beiden deutschen Staaten wahrnehmen. Die stets prekäre Finanzierung der pastoralen, insbesondere aber der diakonisch-caritativen Aktivitäten und Einrichtungen wäre ohne das Engagement der bundesrepublikanischen Schwesternkirchen nicht möglich gewesen. Zwischen 1957 und 1990 fließt innerhalb der evangelischen Kirche eine Summe von 1,4 Milliarden von West nach Ost ${ }^{47}$. Der formelle Weg der evangelischen Kirche von der kirchlichen Einheit trotz staatlicher Teilung und expliziter gesamtdeutscher „Klammer“ zur „besonderen Gemeinschaft“ verhindert nicht ${ }^{48}$, daß das kirchliche Feld der Ort eines informellen Einheitsbewußtseins bleibt und sich hier wie sonst nirgendwo „wie ein Seismograph das Gemeinschaftsbewußtsein und das Auseinanderleben der Menschen im geteilten Deutschland " 49 widerspiegelt. Die katholische Kirche in Dcutschland hat einheitlich in der Hierarchie wie im Laienkatholizismus an der Vorstellung und dem Ziel der Einheit festgehalten und ihre Position auch in einer Phase durchgehalten, als die Interessen der vatikanischen Ostpolitik unter Johannes XXIII. und Paul VI. und der deutschen Kirche auseinanderstreben ${ }^{50}$. Es kommt zwar zur Gründung einer für die DDR zuständigen Berliner Bischofskonferenz, der Berliner Bischofssitz bleibt aber mit seiner Doppelmitgliedschaft die Klammer zwischen beiden Konferenzen und eine endgültige Neuumschreibung der Diözesangrenzen unterbleibt.

\footnotetext{
46 Lange, Pruß, 373.

47 Karl-Heinz Neukamm, Kirchliche Diakonie als notwendige Hilfe zum Leben. Bericht des Diakonischen Werkes der EKD an die Synode, in: cpd-Dokmentation 32 (1991) 2-20, 4.

48 Joachim Meblhausen, Leonore Siegele-Wenschkewitz (Hrsg.), Zwei Staaten - zwei Kirchen? Evangelische Kirche im geteilten Deutschland. Ergebnisse und Tendenzen der Forschung (Leipzig 2000).

49 Claudia Lepp, Die evangelische Kirche als „Klammer“ im geteilten Deutschland. Rollenerwartungen und Rollenwandel 1948 bis 1969, in: Meblhausen, Siegele-Wenscbkewitz, Zwei Staaten $66-84,83$.

50 Ulrich von Hebl, Hans Günter Hockerts (Hrsg.), Der Katholizismus - gesamtdeutsche Klammer in den Jahrzehnten der Teilung? Erinnerungen und Berichte (Paderborn 1996); Karl-Joseph Hummel (Hrsg.), Vatikanische Ostpolitik unter Johannes XXIII. und Paul VI. 1958-1978 (Paderborn 1999). Insbesondere die Aussagen der Zeitzeugen (163-252) geben ein beredtes Zeugnis des Konflikts.
} 
Die binnen weniger Jahrzehnte von einem Mitgliederbestand von über $90 \%$ auf knapp 30\% der Gesamtbevölkerung geschrumpften Kirchen erhalten seit Mitte der achtziger Jahre eine neue Rolle. Die Veränderung geht nicht darauf zurück, daß nun aus einer trägen Volkskirche "gesundgeschrumpfte" Entscheidungskirchen geworden wären. Die gebliebenen Kirchenmitglieder verhalten sich nicht viel anders als ihre Glaubensbrüder im Westen Deutschlands ${ }^{51}$. Die neue Relevanz der Kirchen in der DDR hat einen anderen Ursachenzusammenhang. Sie geht hauptsächlich auf die schnell wachsenden Widersprüche zurück, in die die DDRGesellschaft in den achtziger Jahren unter Modernisierungsdruck gerät. Die kirchliche Gratwanderung zwischen Verweigerung und Anpassung macht sie nun zum (gegen-)institutionellen Dach einer sich neu herausbildenden (Neben- bzw. Gegen-)Öffentlichkeit. Hier können die gesellschaftlichen Widersprüche artikuliert, aus der Verdrängung in die privatisierte Nischen-Gesellschaft herausgelöst und in einem halb-öffentlichen Raum zu Sprache gebracht werden. Die Kirchen werden in die Rolle einer intermediären Kraft hineingedrängt und können sich daraus neue, begrenzte und konfliktreiche Handlungsspielräume eröffnen. In der historisch einmaligen Situation des Umbruchs kommt den Kirchen dann eine gewisse, in ihrer Randlage begründete katalysatorische Wirkung für den durch Außen- wie Innenfaktoren zugleich bedingten Zusammenbruch des DDR-Sytems $\mathrm{zu}^{52}$.

\section{Erklärungsskizze radikaler Entkirchlichung und Säkularisierung}

Wie der europäische Vergleich zeigt, führt die ehemalige DDR diejenige Ländergruppe in Europa an, die den höchsten Grad der Entkirchlichung und den niedrigsten Wert der Religiosität aufweist ${ }^{53}$. Was hat den im Vergleich zu den meisten ost-mitteleuropäischen Ländern so radikalen Entkirchlichungsprozeß im Osten Deutschlands möglich gemacht? Kurt Nowak unterscheidet eine langfristige Erklärungsperspektive, die das überwiegend protestantische Mitteldeutschland seit dem Zeitalter der Reformation in den Blick nimmt, und eine mittlere Sicht, die den Ursachen im Rahmen der Entkirchlichungsprozesse in der ersten Hälfte des 20. Jahrhunderts nachgeht, von einer kurzfristigen Deutung, die sich auf die DDR-Geschichte und die aktive Säkularisierungspolitik des SED-Regimes konzentriert $^{54}$. Nimmt man die längerfristige Perspektive Nowaks auf und erweitert

51 Pollack, Kirche 436ff.; Wolf-Jürgen Grabner, Religiosität in einer säkularisierten Gesellschaft. Eine Kirchenmitgliedschaftsuntersuchung in Leipzig 1989 (Frankfurt a.M. 1994) 61 ff. 52 Pollack, Kirche 446-455.

${ }^{53}$ Gert Pickel, Religiosität und Kirchlichkeit in Ost- und Westeuropa, in: Detlef Pollack, Irena Borowik, Wolfgang Jagodzinski (Hrsg.), Religiöser Wandel in den postkommunistischen Ländern Ost- und Mitteleuropas (Würzburg 1998) 55-85; Miklós Tomka, Paul M. Zulebner, Religion im gesellschaftlichen Kontext Ost(Mittel)Europas (Ostfildern 2000).

${ }^{54}$ Kurt Nowak, Historische Wurzeln der Entkirchlichung in der DDR, in: Heinz Sahner, 
sie um das von Franz Höllinger eingebrachte Argument längerfristiger, mentalitätsgeschichtlicher Einflußprozesse, so legt sich folgende Argumentation nahe. Die Kirchen insbesondere im nördlichen Deutschland tragen eine historische $\mathrm{Hy}-$ pothek: Die in ihrer Geschichte dominierende herrschaftskirchliche Tradition erschwerte die Einwurzelung des kirchlichen Glaubens im Volk. Dort, wo - wie im Norden Deutschlands - die Christianisierung unter den Vorzeichen von Gewalt und kollektiver Taufe vor sich ging, erhielt das Herrschaftsmoment des Glaubens besonders tiefe, mentalitätsgeschichtlich gestützte Wurzeln ${ }^{55}$. Die Konfessionsbildung als zweite, eigentliche Christianisierung in Deutschland vollzog sich in beiden Konfessionen unter der Maxime der engen Bindung von Thron und Alter. Es setzte sich ein Modell von öffentlicher Religion durch, das an die Öffentlichkeit von Herrschaft und Staat anknüpfte. Wo es z. B. früh zur Zurücknahme der Kirchenzucht, des Zusammenspiels von kirchlicher und weltlich-staatlicher Sanktionierung kam, wie in den protestantischen Städten seit Mitte des 18. Jahrhunderts, setzte der Verfall des kirchlichen Lebens lange vor der Industrialisierung $e^{2}{ }^{56}$. Die herrschaftskirchliche Tradition hatte zur Folge, daß in der bürgerlichen Revolution die Religionsfreiheit zur ersten bürgerlichen Freiheit avancierte und die Privatisierung der Religion sich faktisch durchsetzte. Der katholischen Kirche gelang es im 19. Jahrhundert in signifikant stärkerem Maße, vom Modernisierungsprozeß negativ betroffene katholische Bevölkerungsteile an sich zu binden und religiös-kirchlich zu prägen. Für den Protestantismus war der Pfad radikaler Entkirchlichung dort vorgezeichnet, wo die vordem herrschaftsgestützte Religion nicht nur eine Privatisicrung erfuhr, sondern - von der ehemals stützenden Staatsmacht stigmatisiert und bekämpft - aus dem öffentlichen Leben gänzlich ausgeschlossen wurde. Es kann im Grunde nicht überraschen, daß überall dort, wo keine religiös-kirchlichen Wurzeln in einer eigenständigen Volkskultur vorhanden waren - wie neben der DDR auch in Teilen Tschechiens -, es unter der Konstellation einer beinahe 60jährigen antikirchlichen Politik zum radikalen Abbruch kirchlichen Glaubens kam.

Unter welchen Bedingungen - so läßt sich mit Blick auf die Religion als Macht der Lebensführung allgemeiner fragen - nimmt der Modernisierungsprozeß die Gestalt eines „religiösen Supergaus“ 57 an wie im Osten Deutschlands und unter welchen Bedingungen sind alternative Pfade der Religionsentwicklung möglich und wahrscheinlich? Religion wird vom Modernisierungsprozeß dort am negativsten betroffen, wo ihre Privatisierung sich mit einer effektiven, zum Ausschluß

Stefan Schwendtner (Hrsg.), Gesellschaft im Umbruch: 27. Kongreß der Deutschen Gesellschaft für Soziologie (Kongreßband II, Opladen 1995) 665-669.

${ }_{55}$ Franz Höllinger, Volksreligion und Herrschaftskirche. Die Wurzeln religiösen Verhaltens in westlichen Gesellschaften (Opladen 1996) $133 \mathrm{ff}$.

56 Lucian Hölscher, Atlas zur religiösen Geographie im protestantischen Deutschland 18501940 (Bochum 1997, Ms.) 25.

57 Eberbard Tiefensee, Nach dem "religiösen Supergau“ auf dem Weg in cine neue, schwierige "Ökumene“, in: Johannes Röser (Hrsg.), Mehr Himmel wagen, FS Manfred Plate (Freiburg i. Br. 1999) 43-47. 
aus dem gesellschaftlichen Leben führenden Stigmatisierung verbindet. Das ist die Erfahrung des Entkirchlichungsprozesses in Ostdeutschland. Wo die kirchliche Religion auf einen herrschaftlich-politischen Öffentlichkeitsbezug fixiert bleibt, wird sie in der zumindest latenten Gefahr sein, einen Ausweg in der Verbindung von gegenmodernisierenden politisch-nationalen und religiösen Fundamentalismen zu suchen. Auch für diesen Weg lassen sich Beispiele in den national-religiösen Reaktionen in den ehemaligen Ostblockstaaten finden ${ }^{58}$. Wo die moderne Ausdifferenzierung von Religion und Politik nicht gleichzeitig zur Privatisierung der Religion führt, sondern die Religion den Status eines lebendigen Faktors einer zivilgesellschaftlichen Öffentlichkeit erringt, hat sie offensichtlich die besten Entwicklungschancen im Modernisierungsprozeß. Vom konsequenten Ankommen in ziviler Gesellschaft wird die Zukunft von Religion in Deutschland wie im übrigen Europa nicht unwesentlich abhängig sein. Auch ohne politischen Druck zehrt gegenwärtig die radikalisierte Privatisierung der Religion weiter an ihren Wurzeln.

58 José Casanova, Chancen und Gefahren öffentlicher Religion. Ost- und Westeuropa im Vergleich, in: Otto Kallschener (Hrsg.), Das Europa der Religionen (Frankfurt a.M. 1996) $200 \mathrm{ff}$. 